\title{
L'impatto della prematurità sulla qualità di vita del bambino
}

\author{
Mario Eandi ${ }^{(1)}$, Paolo Manzoni (2)
}

\begin{abstract}
In the last years prematurity rates have increased, together with the survival rate among premature subjects. In addition now even infants born at 23 weeks of gestational age can survive. However, some studies demonstrate that premature children are more likely to show late disabilities and to be hospitalised during childhood. In Italy the premature childrens' care level too often depends on the socio-economic status of the Region: central decisions about assistance until the beginning of teenage are yet to be taken. A strict monitoring of pregnancy and of risk factors predisposing to premature birth as well as a careful management of perinatal period should further improve survival and quality of life of these subjects.
\end{abstract}

Keywords: Prematurity; Death in neonatal age; Morbidity during childhood Farmeconomia e percorsi terapeutici 2011; 12(Suppl 3): 25-27

\section{INTRODUZIONE}

In Italia, così come negli altri Paesi, più o meno industrializzati, la prematurità dei nuovi nati, intesa come età gestazionale $<37$ settimane, è in aumento [1,2]. Negli ultimi anni è stato anche registrato un aumento della sopravvivenza dei soggetti prematuri, che ad oggi supera il $90 \%$; contemporaneamente le nuove tecniche di assistenza hanno reso possibile la vita anche ai bambini di sole 23 settimane di età gestazionale o di 500 grammi di peso corporeo [1].

Accanto a queste grandi conquiste, tuttavia, si sono presentate nuove problematiche, prima tra tutte l'elevata incidenza di morbilità e disabilità a lungo termine in questa tipologia di pazienti.

Il presente articolo intende offrire una panoramica sulle principali problematiche che la nascita di un bambino pretermine presenta, in termini di qualità di vita del bambino stesso e dei suoi genitori.

\section{LA PREMATURITÀ E LA QUALITÀ DI VITA DEL BAMBINO}

I bambini prematuri corrono rischi differenti a seconda dell'età gestazionale e del peso corporeo alla nascita.

Nel 2006 in Italia sono nati 517.135 bambini, di cui il 6,5\% prematuri, secondo la seguente ripartizione:

- 5,6\% tra 32 e 36 settimane di età gestazionale;

- $0,7-0,8 \%$ tra 25 e 32 settimane di età gestazionale;

- $0,1-0,2 \%$ tra 22 e 25 settimane $[3,4]$ di età gestazionale.
Oltre il $90 \%$ dei decessi in età neonatale (entro i primi 28 giorni di vita) in Italia colpisce i soggetti nati pretermine; se poi si considera che la maggior parte della mortalità infantile è dovuta proprio a decessi in età neonatale, appare chiaro quanto la prematurità incida sulla sopravvivenza dei bambini [1].

I bambini nati a 23-24 settimane di età gestazionale in effetti sono quelli che presentano le problematiche maggiori: solo $8-25 \%$ di questi sopravvive, e tra i sopravvissuti si registrano tassi del 25\% di disabilità gravi, $25 \%$ di disabilità media e $30-35 \%$ di disabilità lieve.

Bisogna poi anche considerare che i bambini pretermine vanno incontro a più frequenti ospedalizzazioni nel corso della loro infanzia, come testimoniato da alcuni studi, tra cui quelli di McLaurin [5] e Greenough [6].

Lo studio di coorte retrospettivo di McLaurin e colleghi [5] è stato condotto sulla base di dati raccolti da un database delle assicurazioni sanitarie negli USA riguardanti bambini nati a termine $(\mathrm{n}=33.745)$ o pretermine tra la $33^{\circ} \mathrm{e}$ la $36^{\circ}$ settimana di età gestazionale $(\mathrm{n}=1.683)$ nel 2004.

Benché condotto in Stati in cui il sistema sanitario funziona in maniera molto diversa rispetto al nostro, è interessante, e verosimilmente applicabile anche ad altre realtà nazionali, il dato ottenuto circa la spesa sanitaria, risultata di tre volte maggiore per i nati pretermine nell'anno successivo alla dimissione dopo la nascita rispetto ai nati a termine.

Inoltre la percentuale di bambini ospedalizzati è stata inferiore nei bambini a termine rispetto ai pretermine $(7,9 \%$ vs $15,2 \%)$, con una differenza conservata anche suddividendo
${ }^{(1)}$ Cattedra di

Farmacologia Clinica, Facoltà di Medicina e Chirurgia, Università di Torino

${ }^{(2)}$ Divisione di

Neonatologia e TIN, Ospedale S. Anna, Torino
Corresponding author

Mario Eandi

mario.eandi@unito.it 
le ospedalizzazioni in breve (due settimane) e lungo termine (un anno) rispetto alla dimissione dopo la nascita. Anche il numero medio di ospedalizzazioni per bambino e la durata delle degenze sono risultati maggiori nel gruppo dei bambini pretermine (rispettivamente 1,2 vs 1,6 e 3,4 vs 4,5).

Nel primo anno di vita i disturbi respiratori e quelli digestivi sono risultate essere le più frequenti cause di ospedalizzazione, con la bronchiolite da RSV in testa in tutti e tre i sottogruppi, cioè a termine, pretermine con veloce dimissione dopo la nascita e pretermine con tardiva dimissione dopo la nascita (rispettivamente $11,0 \%, 13,1 \%$ e $7,5 \%$ ).

Lo studio di Greenough e colleghi [6] consiste nell'analisi di una coorte di bambini reclutati in uno studio precedente [7], e ha lo scopo di determinare se l'ospedalizzazione per infezione da RSV entro i primi due anni di vita in bambini nati prematuri $(<32$ settimane di età gestazionale) e affetti da broncodisplasia polmonare (BPD) possa determinare in età scolare un eccesso di morbilità e una ridotta funzionalità polmonare, rispetto a coloro che non sono stati ospedalizzati per infezione da RSV. Su 147 bambini sono stati valutati l'utilizzo di risorse sanitarie e i relativi costi, mentre 77 sono stati studiati per determinare la funzione polmonare e l'iper-responsività bronchiale. A 5-7 anni i bambini che erano stati ospedalizzati per infezione da RSV prima dei due anni di età $(n=25)$ avevano costi di trattamento per le prestazioni ambulatoriali significativamente maggiori rispetto a coloro che erano stati ospedalizzati per cause non respiratorie o che non erano stati ospedalizzati $(\mathrm{n}=72)$.

A 8-10 anni 14 bambini che erano stati ospedalizzati per infezione da RSV prima dei due anni di età, rispetto ai 63 soggetti ospedalizzati per cause diverse da RSV, avevano valori significativamente inferiori di:

- $\mathrm{FEV}_{0,75}$ (Forced Expiratory Volume in 0,75 secondi);

- $\mathrm{FEV}_{0,75} / \mathrm{FVC}(\mathrm{FVC}=$ Forced Vital Capacity);

- flussi al 50\% della vital capacity;

- flussi al 75\% della vital capacity.

Quindi in bambini prematuri con BPD l'ospedalizzazione per RSV nei primi due anni di vita si associa a un ridotto calibro delle vie aeree in età scolare, mentre l'iper-reattività bronchiale è risultata comparabile nei gruppi.

\section{LA PREMATURITÀ: ASPETTI SOCIALI}

Numerosi studi hanno dimostrato la correlazione tra basso status economico-sociale e:

- più elevata mortalità perinatale e neonatale [8];

- maggior incidenza di grande prematurità [9];
- minor peso alla nascita [9];

- maggior tasso di ricoveri in terapia intensiva neonatale [9-11];

- maggior morbilità neonatale [11].

Uno studio condotto nell'Irlanda del Nord [12] ha analizzato un database relativo ai ricoveri in terapia intensiva neonatale, rilevando che il sottogruppo di bambini con status socio-economico inferiore ha fatto registrare un tasso di ospedalizzazioni in terapia intensiva neonatale maggiore del 19\% rispetto alla media regionale, un tasso di ricoveri per grande prematurità $(<32$ settimana di età gestazionale) superiore del $18 \%$ rispetto alla media e per scarsa crescita intrauterina del feto del $41 \%$ maggiore rispetto alla media.

Il tasso di ospedalizzazione per nascita multipla è invece risultato maggiore $(45 \%$ in più rispetto alla media) nella fascia di soggetti con status socio-economico più alto. Considerando tutti i bambini in studio, il 34\% delle ospedalizzazioni sono avvenute a causa della prematurità.

Anche in Italia si registrano disparità significative tra regione e regione a livello di assistenza a lungo termine per i neonati prematuri: in effetti, sebbene una raccomandazione del 2008 del Ministero della Salute incoraggi la prestazione di cure e l'assistenza di controllo dei bambini estremamente prematuri fino a 14 anni di età, di fatto un'assistenza a livello centrale non è ancora stata disposta e spetta alle Regioni decidere se destinare fondi e risorse a tale scopo [2]. Al momento non sono previsti supporti economici particolari per le famiglie di bambini prematuri, mentre a livello di diritti sul posto di lavoro è stato stabilito che le madri possono recuperare in seguito i mesi di permesso di maternità di cui non hanno usufruito prima del parto prematuro. Alcuni rappresentanti del Ministero della Salute vorrebbero proporre di prolungare il congedo di maternità di altri 3 mesi al massimo in caso di parto prima delle 32 settimane, ma al momento non ci sono decisioni ufficiali in merito [2].

Una sentenza della Corte Costituzionale del 7 aprile 2011 ha aperto la possibilità per le madri di bambini nati prematuramente e ricoverati di fruire del congedo obbligatorio che spetta loro, o di parte di esso, a partire dalla data d'ingresso del bambino nella casa familiare [13].

Un altro aspetto fondamentale sul quale nelle varie zone d'Italia vi è grande disparità è il coinvolgimento dei genitori nelle cure prestate in ospedale al bambino prematuro [2].

\section{CONCLUSIONI}

L'aumento di prematurità che si sta verificando in molti Paesi industrializzati è da imputare a numerose cause, principalmente all'aumento delle gravidanze gemellari, dell'età materna, e 
delle patologie infettive genito-urinarie femminile (soprattutto Clamidia e Ureaplasma spp), ma esistono altri fattori di rischio che devono essere monitorati attentamente, come schematizzato nella Tabella I [1]. In caso di situazione a rischio di parto pretermine è possibile adottare alcune misure preventive, che consistono principalmente nella riduzione dello stress e nel riposo a letto, ma, a seconda delle condizioni, il management ostetrico-ginecologico si avvale anche di somministrazione di sedativi, tocolitici o antibiotici oppure - in casi estremi e selezionati - fa ricorso al cerchiaggio cervicale.

Fondamentale è la corretta applicazione di protocolli di trattamento prenatale volti a ridurre il rischio di insorgenza di specifiche patologie neonatali legate alla prematurità nel bambino pretermine, e in primis la terapia corticosteroidea e quella antibiotica onde prevenire il distress respiratorio grave e le infezioni connatali [1].

Praticando un adeguato monitoraggio della gravidanza e un'attenta gestione nel periodo perinatale, che esiti in un follow up a lungo termine del neonato prematuro, sarà probabilmente possibile migliorare ulteriormente sopravvivenza e qualità di vita di questo tipo di bambini.

\begin{tabular}{ll}
\hline Precedenti & Precedenti gravidanze con esito in: \\
anamnestici & - decesso fetale o neonatale \\
& - parto pretermine \\
& - complicanze ostetriche \\
& Condizioni di rischio sociale: \\
& - povertà \\
& - denutrizione \\
& - condizioni igieniche precarie \\
& Condizioni di rischio individuale: \\
& - età $<17$ anni \\
& - età $>40$ anni \\
& Familiarità per parti gemellari \\
Cause materne & Gravi malattie della madre persistenti in gravidanza: \\
& - generali \\
& - patologie d'organo \\
& Patologie della gravidanza: \\
& - gestosi \\
& - placenta previa \\
& - distacco di placenta \\
& - rottura prematura precoce delle membrane amniotiche \\
& Gravidanza in età adolescenziale \\
& Rallentata crescita intrauterina \\
& Gemellarità \\
Cause fetali & Patologie funicolari \\
& Malformazioni \\
\hline &
\end{tabular}

Tabella I

Condizioni che ricorrono più frequentemente tra i parti pretermine [1]

\section{BIBLIOGRAFIA}

1. Manifesto dei Diritti del Bambino Nato Prematuro. Disponibile all'indirizzo http://www.vivereonlus.com/ (ultimo accesso maggio 2011)

2. European Foundation for the Care of Newborn Infants (EFCNI). EU benchmarking report 2009-2010. Troppo poco, troppo tardi? Perché l'Europa deve fare di più per i neonati prematuri. Disponibile all'indirizzo http://www.efcni. org (ultimo accesso maggio 2011)

3. Certificato di Assistenza al parto, Analisi dell'Evento nascita - 2006, Ministero della Salute. Disponibile all'indirizzo http://www.salute.gov.it/imgs/C_17_primopianoNuovo_255_documenti_itemDocumenti_0_fileDocumento. pdf (ultimo accesso maggio 2011)

4. Ministero della Salute, http://www.salute.gov.it/imgs/C_17_pubblicazioni_771_allegato.pdf(ultimo accesso maggio 2011)

5. McLaurin KK, Hall CB, Jackson EA, Owens OV, Mahadevia PJ. Persistence of morbidity and cost differences between late-preterm and term infants during the first year of life. Pediatrics 2009; 123; 653-9

6. Greenough A, Alexander J, Boit P, Boorman J, Burgess S, Burke A et al. School age outcome of hospitalisation with respiratory syncytial virus infection of prematurely born infants. Thorax 2009; 64: 490-5

7. Greenough A, Cox S, Alexander J, Lenney W, Turnbull F, Burgess S et al. Health care utilisation of infants with chronic lung disease, related to hospitalisation for RSV infection. Arch Dis Child 2001; 85: 463-8

8. Confidential Enquiry into Maternal and Child Health. Perinatal mortality 2006: England, Wales \& Northern Ireland. London: CEMACH, 2008

9. Smith LK, Draper ES, Manktelow BN, Dorling JS, Field DJ. Socioeconomic inequalities in very preterm birth rates. Arch Dis Child Fetal Neonatal Ed 2007; 92: 11-4

10. Howell EM, Vert P. Neonatal intensive care and birth weight-specific perinatal mortality in Michigan and Lorraine. Pediatrics 1993; 91: 464-9

11. Dolk H (a cura di). Targeting social need: monitoring socioeconomic inequalities in pregnancy outcome. Final report to the Northern Ireland Research and Development Office, 2008

12. Jenkins J, McCall E, Gardner E, Casson K, Dolk H. Socioeconomic inequalities in neonatal intensive care admission rates. Arch Dis Child Fetal Neonatal Ed 2009; 94: F423-F428

13. Sentenza 7 aprile 2011, n.116, Corte Costituzionale. Madri lavoratrici e congedo post partum 